Pontifícia Universidade Católica $_{\text {do Rio de Janeiro }}$

Bernardo Soares de Miranda Carvalho

\title{
A EFICÁCIA DOS CONTROLES DE ENTRADA DE CAPITAIS NO
}

BRASIL

Dissertação de Mestrado

Dissertação apresentada como requisito parcial para obtenção do título de Mestre pelo Programa de PósGraduação em Economia da PUC-Rio.

Orientador: Márcio Gomes Pinto Garcia 

Pontifícia Universidade Católica
do Rio de Janeiro

Bernardo Soares de Miranda Carvalho

\title{
A EFICÁCIA DOS CONTROLES DE ENTRADA DE CAPITAIS NO
}

BRASIL

Dissertação apresentada como requisito parcial para obtenção do título de Mestre pelo Programa de Pós-Graduação em Economia da PUC-Rio. Aprovada pela Comissão Examinadora abaixo assinada.

\author{
Márcio Gomes Pinto Garcia \\ Orientador \\ PUC-Rio
}

Ilan Goldfajn

PUC-Rio

Affonso Celso Pastore AC Pastore \& Associados

João Pontes Nogueira

Coordenador(a) Setorial do Centro de Ciências Sociais - PUC-Rio 
Todos os direitos reservados. É proibida a reprodução total ou parcial do trabalho sem autorização da universidade, do autor e do orientador.

\section{Bernardo Soares de Miranda Carvalho}

Graduado em Economia pela Pontifícia Universidade Católica do Rio de Janeiro no ano de 2002.

Ficha Catalográfica

Carvalho, Bernardo Soares de Miranda

A eficácia dos controles de entrada de capitais no Brasil / Bernardo Soares de Miranda Carvalho ; orientador: Márcio Gomes Pinto Garcia. - Rio de Janeiro : PUC-Rio, Departamento de Economia, 2005.

126 f. ; $30 \mathrm{~cm}$

Dissertação (mestrado) - Pontifícia Universidade Católica do Rio de Janeiro, Departamento de Economia.

Inclui bibliografia

1. Economia - Teses. 2. Controle de capitais. 3. Fluxo de capitais. 4. Elisão de controle de capitais. 5. Eficácia de controle de capitais. I. Garcia, Márcio Gomes Pinto. II. Pontifícia Universidade Católica do Rio de Janeiro. Departamento de Economia . III. Título. 


\section{Agradecimentos}

A Deus.

À minha família e à Isabel Gouvêa Vieira, pelo apoio e incentivo que me deram.

Ao meu orientador, Prof. Márcio Gomes Pinto Garcia, não só pelo fundamental apoio na elaboração desta dissertação, mas também pela significativa contribuição na minha formação acadêmica e profissional. Agradeço especialmente pela confiança depositada ao longo dos vários anos que trabalhamos juntos, desde início da minha graduação até o término do meu mestrado.

Ao Prof. Ilan Goldfajn, pelas importantes intervenções na defesa desta dissertação e pelo extenso aprendizado nos cursos do mestrado e nos ensinamentos diários na Gávea Investimentos.

Ao Prof. Affonso Celso Pastore, pelos excelentes comentários e contribuições na defesa desta dissertação.

Aos professores do Departamento de Economia da PUC-Rio, em especial Afonso Bevilaqua, Dionísio Dias Carneiro, Eduardo Loyo, Juan Pablo Torrez-Martinez, Marcelo de Paiva Abreu, Rogério Werneck e Walter Novaes pelas contribuições na minha formação acadêmica e profissional. Nesta linha quero também agradecer a Armínio Fraga Neto e demais sócios e funcionários da Gávea Investimentos.

A meus amigos da turma do mestrado, pelo apoio e sugestões na elaboração deste trabalho e pelo forte companheirismo ao longo dos pesados dois anos do curso. Em especial, agradeço a Tiago Couto Berriel, Renato Dias de Brito Gomes, Diogo Aquino de Rezende Lopes e Mariano Steinert pelos frutíferos comentários sobre o conteúdo da minha pesquisa.

A Hermann Von Uslar e meus demais amigos da Opus Investimentos, pelo apoio e contribuições que me proporcionaram ao longo da elaboração desta dissertação.

À secretaria do Departamento de Economia da PUC-Rio, em especial à Graça, pela paciência e presteza por todos estes anos.

Ao governo brasileiro, pelo apoio financeiro durante o curso.

E, por fim, mas não menos importante, aos diversos agentes do mercado financeiro que em muito contribuíram para este trabalho ao relatar possíveis operações de elisão dos controles de capital nos anos de 1990 no Brasil. 


\title{
Resumo
}

\author{
CARVALHO, Bernardo Soares de Miranda. A EFICÁCIA dOS CONTROLES
}

DE ENTRADA DE CAPITAIS. Rio de Janeiro, 2005. 126 p. Dissertação de Mestrado - Departamento de Economia, Pontifícia Universidade Católica do Rio de Janeiro.

Esta dissertação analisa a eficácia dos controles de entrada de capitais em restringir e selecionar fluxos financeiros internacionais. A maior contribuição é o foco nas elisões dos controles realizadas pelo mercado financeiro e seus efeitos limitadores na eficácia das restrições ao capital de curto prazo. Primeiro, apresentamos uma abordagem teórica da questão, discutindo como o controle deveria ser implementado. $\mathrm{O}$ modelo tem um arcabouço simples e considera dois tipos de investidores: paciente e impaciente. O primeiro (que se deseja atrair) tem maior interesse no retorno de longo prazo e o segundo (que não se deseja atrair) dá maior valor a ganhos de curto prazo. Mostramos que se o diferencial de juros é endógeno à fixação dos controles, e não há possibilidades de elisão das regulações, então pode ser concebido um mecanismo de taxação à entrada de capitais que induz à opção de investir a longo prazo para ambos os investidores, na ausência de crises de confiança. Se vier a se realizar uma probabilidade de default maior que a esperada pelo agente, o investimento será revertido, a não ser que seja oferecido um maior diferencial de taxa de juros. Controles ex-post de saída de capitais também poderiam evitar a reversão do investimento, entretanto, nós não consideramos esta possibilidade devido à grande perda de reputação que ela acarreta. Se o diferencial de taxa de juros for exógeno, como é tipicamente o caso relevante no Brasil, podemos ter um incentivo ao investidor disfarçar seu capital como se fosse de longo prazo mesmo quando já espera sacar no curto prazo. Prosseguimos a análise focando o impacto da capacidade de elisão dos controles pelo mercado. Incorporamos no modelo a elisão às restrições de entrada, mostrando que isto elimina a eficácia dos controles de capitais. Em verdade, as taxas do controle de capitais são determinadas pelo mercado, e então, quanto mais desenvolvido e sofisticado o mercado financeiro do país for, mais ativos substitutos e operações de engenharia financeira podem ser usados para circundar os controles. Fundamentamos nossas conclusões relatando diversas operações de elisão de controles de capitais dos anos noventa, que deixam claro a dificuldade de implementar de facto os controles de capitais. Terminamos a dissertação com uma análise econométrica sobre a duração da eficácia dos controels, via a estimação funções de reposta a impulso. Os resultados indicam que os controles de capitais têm apenas entre dois a seis meses de eficácia em restringir os influxos de capitais financeiros, corroborando a análise dos casos relatados de elisão dos controles.

\section{Palavras-chave}

Controles de capitais, fluxos de capitais, elisão de controles de capitais, eficácia de controles de capitais 


\section{Abstract}

CARVALHO, Bernardo Soares de Miranda A EFICÁCIA DOS CONTROLES

DE ENTRADA DE CAPITAIS. Rio de Janeiro, 2005. 126 p. Ms Dissertation o Departamento de Economia, Pontifícia Universidade Católica do Rio de Janeiro.

This thesis analyses the effectiveness of capital inflows controls in changing the size and composition of international financial flows. The major contribution is the focus on capital controls circumvention by the financial market and its limiting impacts on the effectiveness of short-run capital flows restrictions. At first, it is presented a theoretical approach to the question, discussing how it should be implemented. The model has a simple framework and considers two types of financial investors: patient and impatient. The first is more interested in long run returns, and the last gives more value for short run gains. We show that if the interest rate differential is endogenous to the controls fixation, and there is no possibility of regulations' circumvention, then could be arranged a capital entrance tax scheme that induces the long run investment option for both investors, in absence of a confidence crisis. In occurrence of a higher probability of default than expected by the agent, the investment will be reverted unless higher interest rate differential is provided. Ex-post outflows capital controls could also avoid the investment reversion, nevertheless, we do not consider it here. If the interest rate differential is exogenous, which is typically the relevant case in Brazil, we might see an incentive to the investor to rename its capital as long run even when she expects to reverse the investment in the short run. We continue the analysis focusing the impact of the capacity of capital controls elision by the market. We incorporate in the model the circumvention of the entrance restrictions, showing that it ruins the capital controls effectiveness. In fact, the taxes of the capital controls are really determined by the market, and therefore, as more developed and sophisticated the financial market of the country is, more substitute assets and engineer financial operations can be used to circumvent the control. We underpin our conclusions describing several capital controls elisions operations in Brazil during the nineties, which make clear the difficulties of capital controls de facto implementation. We finish the dissertation with an impulse response function analyses, that indicates that the capital controls have only between two to six months of effectiveness in restricting financial capital inflows.

\section{Keywords:}

Capital Controls, Capital Flows, capital controls circumventions, capital controls' effectiveness 


\section{Sumário}

1 INTRODUÇÃO

2 MODELO 15

2.1. MODELO ORIGINAL 17

2.2. MODELO COM ELISÃO DOS CONTROLES DE
ENTRADA CAPITAIS

2.2.1. Modelo com Função Custo de Elisão de Controles de

2.2.2. Modelo com custo de elisão de controles de capitais

$\begin{array}{ll}\text { como função das taxas oficiais } & 48\end{array}$

2.3. CONCLUSÃO DOS MODELOS TEÓRICOS 53

3 CONTROLES DE CAPITAIS NO BRASIL 58

3.1. CASOS DE ELISÃO DE CONTROLES DE ENTRADA
DE CAPITAIS NO BRASIL

3.1.1. CONCLUSÃO DOS CASOS DE ELISÃO 89

4 UMA ANÁLISE VIA VAR DA EFICÁCIA DOS CONTROLES

DE ENTRADA EM REDUZIR O INFLUXO DE CAPITAIS 95

5 CONCLUSÃO 109

6 BIBLIOGRAFIA 112

7 APÊNDICE 1: Controles de Capitais no Chile 116

8 APÊNDICE 2: Modelo com Função Custo de Elisão de
Controles de Capitais côncava

9 APÊNDICE 3: Legislação de Controles de Capitais 1996-2004 119 


\section{Lista de llustrações}

GRÁFICO 1: Modelo 1 - Retorno Esperado de longo prazo 24

GRÁFICO 2: Modelo 1 - Retorno Esperado de curto prazo 25

GRÁFICO 3: Modelo 1 - Curvas de Indiferença entre LP e Ext 28

GRÁFICO 4: Modelo 1 - Curvas de Indiferença entre CP e Ext 30

GRÁFICO 5: Modelo 1 - Curvas de Indiferença entre sacar ou não em t = 132

GRÁFICO 6: Modelo 1 - Solução gráfica. 33

GRÁFICO 7: Modelo 1 - Determinação de M e $\tau$ correspondentes 34

GRÁFICO 8: Modelo 1 - Curvas de Indiferença entre CP, LP e Ext 35

GRÁFICO 9: Modelo $1-$ Exemplo com $\left(i-i^{*}\right)=15 \%$

GRÁFICO 10: Modelo 2 - Custo de Elisão Constante - Tipo Paciente 52

GRÁFICO 11: Modelo 2 - Custo de Elisão Constante - Tipo Impaciente. 52

GRÁFICO 12: Modelo 2 - Função Custo de Elisão linear - Tipo Paciente. $\quad 55$

GRÁFICO 13: Modelo 2 - Função Custo de Elisão linear - Tipo Impaciente. 55

GRÁFICO 14: Modelo 2 - Solução Implícita com elisão 57

GRÁFICO 15: Controles - Entrada de Investimento em Carteira 67

GRÁFICO 16: Controles - Índice de Controles de Entrada de Capitais (ICC) 70

GRÁFICO 17: Controles - Índice de Controles de Saída de Capitais (ICC-S) 71

GRÁFICO 18: Controles - Movimento via CC-5 e CIPD 92

GRÁFICO 19: VAR - Restrições à entrada de capitais 102

GRÁFICO 20: VAR - Função de Resposta a impulso 1 a ICC 106

GRÁFICO 21: VAR - Função de Resposta a impulso 2 a ICC 109

GRÁFICO 22: VAR - Função de Resposta a impulso 2 a Influxo de Capital 109

GRÁFICO 23: VAR - Função de Resposta a impulso 3 a ICC 112

GRÁFICO 24: VAR - Função de Resposta a impulso 2 a Fluxo de Capital 112

GRÁFICO A2.1: Apêndice 2 - Solução Tipo Paciente 119

GRÁFICO A2.2: Apêndice 2 - Solução Tipo Impaciente 119

GRÁFICO A2.3: Apêndice 2 - Solução implícita com elisão 119

FIGURA 1: Modelo - Árvore de Possibilidades 18

FIGURA 2: Controles - Caso de Elisão \#3 80

FIGURA 3: Controles - Caso de Elisão \#3 80

FIGURA 4: Controles - Caso de Elisão \#3 81

FIGURA A1.1: Apêndice 1 - Taxa equivalente no Chile 118

TABELA 1: Controles - Composição Carteira Anexo IV 94

TABELA 2: VAR - Estimação VAR $01 \quad 101$

TABELA 3: VAR - Estimação VAR $02 \quad 104$

TABELA 4: VAR - Estimação VAR 03 